\title{
Prevalence of oral candida albicans infection in HIV sero-positive patients in Abakaliki
}

\author{
Okonkwo E. C. ${ }^{1}$, Alo M. N. ${ }^{2}$, Nworie O. ${ }^{2}$, Orji J. O. ${ }^{1}$, Agah M. V. ${ }^{1,}$ " \\ ${ }^{1}$ Department of Applied Microbiology, Faculty of Biological Sicences, Ebonyi State University, Abakaliki, Ebonyi State, Nigeria \\ ${ }^{2}$ Department of Biological Sciences, Faculty of Science and Technology, Federal University Ndufu Alike-Ikwo, Ebonyi State, Nigeria
}

\section{Email address:}

mosesalo@yahoo.co.uk(Alo M. N.)

\section{To cite this article:}

Okonkwo E. C. , Alo M. N., Nworie O. , Orji J. O., Agah M. V.. Prevalence of Oral Candida Albicans Infection in HIV Sero-Positive Patients in Abakaliki. American Journal of Life Sciences. Vol. 1, No. 2, 2013, pp. 72-76. doi: 10.11648/j.ajls.20130102.18

\begin{abstract}
Oral thrush is commonly associated with HIV infection. The causative agent is a yeast strain that is originally a commensal of the oral cavity. Most species of the genus Candida that causes oral candidasis in HIV patients if not properly identified and treated with the drug of choice could result in resistant to the drugs and make treatment very difficult. This study was carried out to establsish the species spectrum of the common yeast(Candida albicans) associated with oral candidiasis in HIV patients on antiretroviral treatment in Abakaliki. A total of 240 samples were collected from HIV sero-positive males(64) and females(176) at the two hospitals. 40 control samples from HIV sero-negative persons were also collected. The samples were cultured on Sabouraud dextrose agar and Candida species were isolated and characterized using germ tube test and sugar fermentation tests. Out of the 240 subjects(HIV sero-positive patients) examined for oral candidiasis, the carriage rate of oral candidiasis were $12.5 \%(30 / 240)$. Candida albicans accounted for $80.00 \%$ in HIV sero-postive patients, followed by Candida pseudotropicalis(10.0\%). More women, 21(8.75) had oral candidiasis than men $9(3.75 \%)$. HIV patients whether or not on drugs were predisposed to oral candidiasis. C. albicans $(76.19 \%)$ is the commonest species associated with HIV infected patients on ART(Active Retroviral Therapy) followed by Candida pseudotropicalis(14.29\%), Candida tropicalis(4.76\%) and Candida parapsilosis(4.76\%). Among the patients not on ART Candida albicans(88.89\%) was most prevalent, followed by Candida guilliermondii(11.11\%). C. albicans still remains the leading cause of oropharyngeal candidiasis in HIV infected persons within the study population. Constant identification of isolates of yeasts infecting HIV infected persons and the immune compromised will further enhance the appropriate treatment and minimize the spread emergence of antifungal resistance.
\end{abstract}

Keywords: Prevalence, Oral Candidiasis, HIV Sero-Positve Patients, ART, Abakaliki

\section{Introduction}

Oral candidiasis(also known as oral thrush) is a common opportunistic mycosis(yeast infection) of Candida species on the mucous membranes of the mouth(1-2). C. albicans is the most common species of yeast isolated from patients with oral candidiasis(3). The incidence of opportunistic infections due to Candida albicans and other Candida species has been increasing(4). Oral Candidiasis is the most common HIV related oral lesion and most patients are infected with a strain originally present as a commensal of the oral cavity(5).

The spectrum of Candida infection is diverse, starting from asymptomatic colonization to pathogenic forms. The low absolute CD4+ T-lymphocyte count has traditionally been cited as the greatest risk factor for the development of oropharyngeal candidiasis and current guidelines suggest increased risk once CD4+ T lymphocyte counts fall below 200 cells/ $\mu(6)$.

The first step in the development of a candidal infection is colonization of the mucocutaneous surfaces(6). HIV infection is not only associated with increased colonization rates but also with the development of overt disease. During the course of HIV infection, the rate of Candida infection is inversely related to the CD4 counts of the patient which in turn depends on the use of Anti-retroviral treatment(7). HIV-positive patients carry more and a greater variety of yeasts than HIV-negative subjects. The prolonged management of oral candidiasis in HIV patients might cause the development of drug resistance candidiasis(8). Although the introduction of antiretroviral therapy(ART) has had a major impact on the infectious complications of $\operatorname{AIDS}(9)$, Candidiasis still remains a 
common opportunistic infection in HIV-infected patients(6). Hence this present study is aimed to determine the prevalence of oral Candida albicans infection in HIV positive patients in Abakaliki.

\section{Materials and Methods}

\subsection{Study Population}

Two hundred ad fourty(240) HIV seropositive males and females within the age range of 19-70 years receiving antiretroviral therapy at Federal Teaching Hospital Abakaliki II(FETHA II) and General Hospital Ezzamgbo were recruited for this study, fourty(40) control samples from HIV seronegative persons were also collected.

\subsection{Sample Collection}

Two hundred ad fourty(240) samples were obtained from the oral cavities of HIV-positive patients(men, 64; women, 176) with clinically important lesions of oral candidiasis receiving antiretroviral therapy at Federal Teaching Hospital Abakaliki II(FETHA II) and General Hospital Ezzamgbo(GHE). Fourty(40) samples were also collected from HIV seronegative persons, which served as control. Informed consent was obtained from participants and procedures were performed according to FETHA II and GHE board of ethical committee. The oropharyngeal candidiasis(OPC) lesions in $\mathrm{HIV}$-infected patients were diagnosed by an infectious diseases specialist on the basis of clinical presentation, findings of direct microscopic examination, and positive culture results.

\subsection{Isolation of Candida albicans and Other Clinical Important Candida species}

Specimens were obtained from the OPC lesions of the tongue or the buccal mucosa by using sterile cotton swabs. These swabs were incubated in Sabouraud's dextrose agar with choramphenicol(Merck, Germany) at $32^{\circ} \mathrm{C}$ for 48 hours(under aerobic conditions) and in CHROMagar ${ }^{\mathrm{TM}}$ Candida(CHROMagar, France) at $35^{\circ} \mathrm{C}$ for 48 hours(in the dark) for production of species-specific colors.

Various chromogenic culture media capable of distinguishing C. albicans from other clinically important yeast strains are commercially available. Such media distinguish Candida strains from other yeast strains on the basis of the color changes produced by the Candida colonies, which are measured using $\mathrm{pH}$ indicators and by fermentation of specific compounds or chromogenic substrates for the presumptive identification of C. albicans, C. pseudotropicalis, C. tropicalis, C. parapsilosis and C. guilliermondii(10). We used a $10 \% \mathrm{KOH}$ preparation and Giemsa stain for microscopic examination of pseudohyphae and yeast cell forms. We used sugar fermentation and sugar assimilation tests with RapID ${ }^{\mathrm{TM}}$ Yeast Plus System(Remel, USA) according to the manufacturer's instructions. Fresh yeast colonies were incubated with human serum at $37^{\circ} \mathrm{C}$ for 3 hours to test for germ tube formation. Development of filamentous-form cells and chlamydospore formation were evaluated by culturing the yeast isolates on Dalmau plates(cornmealTween 80 agar) at $30^{\circ} \mathrm{C}$ for 48 hours(11).

Table 2. Gram tube formation, sugar fermentation and assimilation of Candida species isolated from HIV positive patients in Abakaliki

\begin{tabular}{|c|c|c|c|c|c|c|c|c|c|c|c|}
\hline \multirow{2}{*}{$\mathbf{S} / \mathbf{N}$} & \multirow{2}{*}{$\begin{array}{c}\text { Growth on } \\
\text { SDA }\end{array}$} & \multirow{2}{*}{$\begin{array}{c}\text { Germ tube } \\
\text { test }\end{array}$} & \multirow{2}{*}{ Pseudohyphae } & \multicolumn{4}{|c|}{ Sugar assimilation } & \multicolumn{4}{|c|}{ Fermentation } \\
\hline & & & & Gly & Suc & Lac & Gal & Gly & Suc & Lac & Gal \\
\hline 1 & + & - & + & + & + & - & + & + & + & - & + \\
\hline 2 & + & - & + & + & - & + & + & + & + & + & + \\
\hline 3 & + & + & + & + & - & + & - & + & + & + & + \\
\hline 4 & + & + & + & + & + & + & - & + & - & - & - \\
\hline 5 & + & - & + & + & + & - & + & + & + & + & - \\
\hline 6 & + & - & + & + & - & + & - & + & - & + & - \\
\hline 7 & + & - & + & + & + & - & + & + & - & - & + \\
\hline 8 & + & + & + & + & + & - & + & + & + & - & + \\
\hline 9 & + & + & + & $\sqrt{ }$ & $\sqrt{ }$ & $\sqrt{ }$ & $\sqrt{ }$ & $\sqrt{ }$ & $\sqrt{ }$ & $\sqrt{ }$ & $\sqrt{ }$ \\
\hline
\end{tabular}

Key: $+=$ Positive, - = Negative

\section{Results}

Out of the two hundred and fourty(240) samples of HIV positive patients on ART collected from FETHA II and GHE, 176 were from women and 64 were from men. The highest numbers of men screened were between the age ranges of 26-55(40), followed by 36-45(14), 15-25(6), and 45-55(2), while age range of 56-65(1) and 66-75(1) were the lowest screened. The highest number of female screened were within the age range of 26-35(125), followed by 36-45(75), 15-25(26), 46-55(8), and 56-65(4), while $66-75(2)$ were the lowest screened.

The result of the Gram tube formation, fermentation and assimilation of Candida species isolated from HIVseropositive patients is shown in Table 2 below

Out of the 30 Candida species isolated, 24(80.00\%) were Gram tube formers and were identified as Candida albicans. The non albicans species identified were Candida tropicalis 1(3.33\%), Candida pseudotropicalis 3(10.00\%), Candida parasiolosis $1(3.33 \%)$, and Candida guilliermondii $1(3.33 \%)$ (Table 3). 
Table 3. Species of Candida obtained from 240 HIV infected patients attending Federal Teaching Hospital Abakaliki II (FETHA II) and General Hospital Ezzamgbo (GHE)

\begin{tabular}{lcc}
\hline Species of Candida & $\begin{array}{c}\text { Number of } \\
\text { Isolate }\end{array}$ & $\begin{array}{c}\text { Percentage of } \\
\text { Isolate (\%) }\end{array}$ \\
\hline Candia albicans & 24 & 80.00 \\
Candida pseudotropicalis & 3 & 10.00 \\
Candida parapsilosis & 1 & 3.3 \\
Candida tropicalis & 1 & 3.3 \\
Candida guilliermondii & 1 & 3.3 \\
Total & 30 & 100 \\
\hline
\end{tabular}

Candida albicans was the most prevalent species isolated from both HIV seropositive subjects on ART 17(76.19\%) and that not on ART $8(88.89 \%)$. Other species recovered from patients on ART were, Candida pseudotropicalis 3(14.29\%), Candida tropicalis $1(4.76 \%)$, and Candida parasilosis $1(4.76 \%)$. Candida guilliermondii $1(11.11 \%)$ was the only non-albicans species found in patients not receiving ART(Table 4).

Table 4. Candida species distributed in HIV positive and HIV negative persons both on ART and not on ART

\begin{tabular}{llcc}
\hline Subject & Species & $\begin{array}{c}\text { Number of } \\
\text { isolates }\end{array}$ & $\begin{array}{c}\text { Percentage of } \\
\text { isolates (\%) }\end{array}$ \\
\hline \multirow{3}{*}{$\begin{array}{l}\text { Isolates on } \\
\text { ART }\end{array}$} & Candida albicans & 16 & 76.19 \\
& Candida & 3 & 14.29 \\
Isolates not & Candida tropicalis & 1 & 4.76 \\
on ART & Candida parapsilosis & 1 & 4.76 \\
\hline
\end{tabular}

\section{Discussion}

The incidence of opportunistic infections due to Candida albicans and other Candida spp. has been increasing(4). Candida albicans is one of the most frequently isolated yeasts in clinical laboratories from HIV/AIDS patients(12). Studies have shown that this organism can account for up to $75 \%$ of the yeasts recovered from sites of infection(13). Rapid identification of candidiasis is important for the clinical management of immunocompromised patients(4). During the course of HIV infection, the rate of Candida infection is inversely related to the CD4 counts of the patient which in turn depends on the use of Anti-retroviral treatment(6).

Table 1. Age and sex distribution of HIV positive patients on ART at Federal Teaching Hospital Abakaliki II (FETHA II) and General Hospital Ezzamgbo (GHE)

\begin{tabular}{cccc}
\hline $\begin{array}{c}\text { Age Group } \\
\text { (years) }\end{array}$ & $\begin{array}{c}\text { Number of Male } \\
\text { Screened }\end{array}$ & $\begin{array}{c}\text { Number of Female } \\
\text { Screened }\end{array}$ & Total \\
\hline $15-25$ & 6 & 20 & 26 \\
$26-35$ & 40 & 85 & 125 \\
$36-45$ & 14 & 61 & 75 \\
$46-55$ & 2 & 6 & 8 \\
$56-65$ & 1 & 3 & 4 \\
$66-75$ & 1 & 1 & 2 \\
Total & 64 & 176 & 240 \\
\hline
\end{tabular}

The numbers of women screened in this study were found to be 176 , while men 64 (Table 1). This is probably because most men rarely go for routine checkup, until the disease has reached symptomatic stage. Among the patients examined only three reported having pain when swallowing food and having altered taste, this suggested that oral thrush candidiasis in most cases asymptomatic.

The result of this study provides evidence of the isolation of Candida species in the oral pharyngeal candidiasis(OPC) of HIV infected persons on Antiretroviral Therapy(ART) as well as in those not on ART in Abakaliki. The current isolation rate of $C$. albicans in HIV seropositive patients was $80.00 \%$ which is close to the $82.2 \%$ reported from $\operatorname{Iran}(4), 71.25 \%$, India(6), but higher than $61.8 \%$ reports from Ethiopia(5), 58.3\%, Mexico(14) and Sao Paulo, Brazil(66.4\%) (15). The non albicans species identified were Candida pseudotropicalis (10.00\%), Candida tropicalis (3.33\%), Candida parasiolosis $(3.33 \%)$, and Candida guilliermondii $(3.33 \%)$ (Table 3). Non-albicans Candida as an agent of oral candidiasis in HIV/AIDS patients is documented $(16,14)$. Although $C$. albicans is the most frequently implicated pathogen in OPC, other Candida spp. are being increasingly associated with invasive candidiasis(17).

HIV seropositive people whether or not on ART are predisposed to oral candidiasis. This does not agree with most studies, (18) reported that following the introduction of highly active antiretroviral therapy(HAART) there was reduction in occurrence of opportunistic infections, prevalence of oral manifestation and oral candidiasis. Arribas et al. (19) also suggested that the reduction in the frequency of oral candidiasis was only related to immunological improvement after introduction of antiretroviral therapy including protease inhibitor( $\mathrm{P} 1)$, which increase the number of CD4+ cells. However, some HIV positive patients with relatively high CD4+ cell counts develop oral candidiasis(6). Control samples obtained from HIV seronegative persons in this study showed growth on Sabaraud's dextrose agar (SDA), revealing Candida albicans (88.89\%) and Candida guilliermondii (11.11\%) (Table 4). This is in line with the report of Hamza et al. (8) that Candida species can be found in both HIV positive and HIV negative persons with a significant difference in oral carriage of the organism.

The carriage rate of oral candidiasis in HIV seropositive people in Abakaliki is $12.5 \%$ (30/240). This rate is higher than the $9.68 \%$ reported by Lar et al. (20) from HIV seropositive people in Jos Nigeria, but lower than the $34.4 \%$ reported by Enwuru et al., (21) from seropositve people in Lagos Nigeria. Agwu et al. (22) in Uganda found Candida species in 52\% of their subjects who had HIV and were on antifungal therapy. The carrier rate was also lower than in studies from Thailand, Adults $66.6 \%$ and children $70 \%$ (23-24). Hongkong(54.8\%) (25), Italy(61.9\%) (26), Germany(73.8\%) (27) and India(65.3\%) (28). The low prevalence of oral Candida in this study may be attributed to patient strict adherence to their antiretroviral regimen. 
However, Candida guilliernmondii was not present in HIV patients on ART on ART but was isolated in patients not on ART although Candida albicans still remains the leading cause of oral and vagina candidiasis in HIV infected person. Care must be taken in identifying species especially amongst persons that are immunecompromised, in order to guide treatment properly.

Various Candida species causing oral candidiasis exist in about $12.5 \%$ HIV/AIDs infected patients attending Federal Teaching Hospital Abakaliki II(FETHA II) and General Hospital Ezzamgbo(GHE). Routine checks for opportunistic infections including oral candidiasis are important and should be carried out at intervals to help monitor disease progression and also prevent subsequent complications such as candidemia. Identifying Candida to its species level is important because it helps guiding proper treatment.

\section{References}

[1] William JD and Timothy B.G. 2006. Andrews' diseases of the skin: Clinical dermatology. Saunders Elsevier. P. 45. ISBN 0-7216-2921-0.

[2] Crispian S. Oral and maxillofacial medicine: The basis of diagnosis and treatment(2nd ed. ed. ). Edinburgh: Churchill Livingstone 2008 PP. 191-199.

[3] Fidel PL. Candida-host interactions in HIV disease: Relationships in oropharyngeal candidiasis. Adv Dent Res 2006, 19: 80-84.

[4] Mousavi SAA, Salari S, Rezaie S, Nejad NS, Hadizadeh S, Kamyabi H \& Aghasi H. Identification of Candida species isolated from oral colonization in Iranian HIV-positive patients, by PCR-RFLP method. Jundishapur J Microbiol 2012, 5(1): 336-340. DOI: 10.5812.

[5] Wabe NT, Hussein J, Suleman S. \& Abdella K. In vitro antifungal susceptibility of Candida albicans isolates from oral cavities of patients infected with human immunodeficiency virus in Ethiopia. Journal of Experimental and Integrative Medicine 2011, 1(4): 265-271.

[6] Khan AP, Malik A \& Khan SH.. Profile of candidiasis in HIV infected patients Iranian Journal of Microbiology 2012, 4(4): 204-209.

[7] Pappas PG, Rex J.H. \& Lee J. A prospective observational study of candidemia: epidemiology, therapy, and influences on mortality in hospitalized adult and pediatric patients. Clin Infect Dis 2003, 37(5): 634-643.

[8] Hamza OJM, Mate M, Kikwilu E, Moshi E, Mugust J, Mikke F, Verius M \& Vander A. Oral manifestation in HIV infection in children and adults ecieving HAART in Dar Salaam, Tanzania. Bioned Central Oral Health 2008, 6:12.

[9] Michelet C, Arvieux C, François C, Besnier JM, Rogez JP, Breux JP \&and Souala F. Opportunistic infections occurring during highly active antiretroviral treatment. AIDS 1998 12: 1815-1822.
[10] Moris D, Melhem M, Martins M \& Mendes R. Oral Candida spp. colonization in human immunodeficiency virus-infected individuals. J. Venom. Anim. Toxins 2008, 14(2): 224-257.

[11] Katiraee F, Khosravi AR, Khalaj V, Hajiabdolbaghi M, Khaksar A \& Rasoolinejad M. Oropharyngeal candidiasis and oral yeast colonization in Iranian Human Immunodeficiency Virus positive patients. J Med Mycol 2010, 20(1): 8-14.

[12] Mirhendi S, Kordbacheh P, Pezeshki M \& Khorramizadeh M. Simple and rapid identification of most medically important Candida species by a PCR-restriction enzyme method. Acta Medica Iranica 2003, 41: 2.

[13] Espinel-Ingroff A, Brachiesi F, Hazen KC, MartinezSuarez JV \& Scalise G. Standardization of antifungal susceptibility testing and clinical relevance. Med Mycol 1998, 36: 68-78

[14] Sanchez-Vargas LO, Ortiz-Lopez NG, Villar M, Moragues MD, Aguirre JM, Cashat-Cruz M, Lopez-Ribot JL, GaitanCepeda LA \& Quindos G. Oral Candida isolates colonizing or infecting human immunodeficiency virus-infected and healthy persons in Mexico. Clin Microbiol J 2005, 43: 4159-4162.

[15] Ito CY, de Paiva MCA, Loberto JC, dos Santos SS \& Jorge AO. In vitro antifungal susceptibility of Candida spp. isolates from patients with chronic periodontitis and from control patients. Braz Oral Res 2004, 18:80-84.

[16] Pruthvi BC, Vikram S, Suman SK, Jayaprakash B. \& Rau NR. Spectrum of Clinical Presentation and Opportunistic Infections in HIV: An Indian Scenario, 13th International Congress on Infectious Diseases 2006, P. e484

[17] Shokohi T, Bandalizadeh Z, Hedayati MT \& Mayahi S. In vitro antifungal susceptibility of Candida species isolated from oropharyngeal lesions of patients with cancer to some antifungal agents. Jundishapur J Microbiol 2011, 4(1): S19S26.

[18] Cassone A, De Bernardis F, Torosantucci A, Tacconelli E, Tumbarello M \& Cauda $\mathrm{R}$ In vitro and in vivo anticandidal activity of human immunodeficiency virus protease inhibitors. J Infect Dis 1999, 180, 448-453.

[19] Arribas JR, Hernandez-albujar S, Gonzales-garcia JJ, Peña JM, Gonzáles A, Cañedo T, Madero R, Vazquez JJ \&Powderly WG. Impact of protease inhibitor therapy on HIV-related oropharyngeal candidiasis. AIDS 2000, 14: 979-85.

[20] Lar PM, Pam KV, Tiri Y, Olukose S, Yusuf A, Dashen MM \& Mawak JD. Prevalence and distribution of Candida Species in HIV infected persons on antiretroviral therapy in Jos. Journal of Medicine and Medical Science 2012, 3(4): 254-259

[21] Enwuru CA, Ogunledun A, Ogbonna F, Enwuru NV, Aneidobe $\mathrm{M} \&$ Adeiga A. Fluconazole resistant opportunistic oropharyngeal Candida and non- Candida yeast-like isolates from HIV infected patients attending ARV clinics in Lagos, Nigeria. J Afr Health Sci 2008, 18(3): 142-148.

[22] Agwu E, Ihongbe JC, McManus BA, Moran GP, Coleman DC \& Sullivan DJ. Distribution of yeast species associated 
with oral lesions in HIV-infected patients in Southwest Uganda. Med Mycol 2011, 12.

[23] Teanpaisan R \& Nittayananta W. Prevalence of Candida species in AIDS patients and HIV-free subjects in Thailand. J Oral Pathol Med 1998, 27: 4-7.

[24] Pongsiriwet S, Iamaroon A, Sriburee P, Pattanaporn K \& Krisanaprakornkit S. Oral colonization of Candida species in perinatally-HIV infected children in northern Thailand. J Oral Sci 2004, 46: 101-105.

[25] Tsang CS \& Samaranayake LP. Oral yeasts and coliforms in HIVinfected individuals in Hong Kong. Mycoses 2000, 43: 303-308.
[26] Campisi G, Pizzo G, Milici ME, Mancuso S \& Margiotta V. Candida carriage in the oral cavity of human immunodeficiency virusinfected subjects. Oral Surg Oral Med Oral Pathol 2002, 93: 281-286.

[27] Schmidt-Westhausen A, Schiller RA, Pohle HD \& Reichart PA. Oral Candida and Enterobacteriaceae in HIV-1 infection: correlation with clinical candidiasis and antimycotic therapy. J Oral Pathol Med 1991, 20: 469-472.

[28] Gugnani HC, Becker K, Fegeler W, Basu S, Chattopadhya D, Baveja U, Satyanarayana S, Kalghatgi T \& Murlidhar A. Oropharyngeal carriage of Candida species in HIV-infected patients in India. Mycoses 2003, 46: 299-306. 